used to measure pro- and anti-inflammatory cytokines. Viral replication was investigated using a plaque assay technique and by directly infecting CRLM tissue with a related GFP-encoding VV. To demonstrate induction of the innate immune response we infected PBMCs with the virus and measured levels of degranulation of Natural Killer (NK) cells, and the cytotoxic ability when activated PBMCs were exposed to CRC targets.

Results JX-594 treatment results in up to $75 \%$ lysis of CRC cell lines in $96 \mathrm{~h}$. VV can replicate in CRC cells (250-fold replication in $72 \mathrm{~h}$ ), thus potentially spreading through a tumour cell population, magnifying its anti-tumour effect. VV is able to produce large quantities of GMSCF. This is a cytokine that can stimulate a variety of immune effector cells including dendritic cells, which can be recruited to the tumour environment. In our study, more than 50\% of the NK cells become "armed" in response the viral treatment, with the release of granyzmes that can induce apoptosis of target tumour cells. These activated NK cells when exposed to CRC targets, result in a $40 \%-50 \%$ lysis of the tumour cells in $24 \mathrm{~h}$. VV treatment can also cause the up-regulation pro-inflammatory cytokines around the tumour environment, and potentially suppress new vessel formation by inhibiting VEGF.

Conclusion Viral therapeutics holds promise as a novel treatment modality for treatment of disseminated malignancy, providing direct tumour-specific lysis and the induction of tumour-specific innate immunity. It has been shown to be safe for intravenous delivery and early studies show promising results. We propose that VV may provide an adjunct to liver resection for treatment of liver cancer, and we will soon embark on a multi-centre early phase trial, whereby VV will be administered intravenously pre-operatively.

Competing interests None declared.

\section{OC-057 10 YEARS ON FROM THE IMPROVING OUTCOMES GUIDANCE-DEVELOPMENT OF A TERTIARY PANCREATIC CANCER UNIT}

doi:10.1136/gutjnl-2012-302514a.57

${ }^{1} \mathrm{M}$ Johnstone, ${ }^{* 1,2} \mathrm{C} \mathrm{M}$ Halloran, ${ }^{1,2} \mathrm{P}$ Ghaneh, ${ }^{1,2} \mathrm{R}$ Sutton, ${ }^{1,2} \mathrm{~J} \mathrm{P}$ Neoptolemos, ${ }^{2}$ M G T Raraty. 'Liverpool NIHR Pancreas Biomedical Research Unit, University of Liverpool, Liverpool, UK; ${ }^{2}$ Royal Liverpool University Hospital, Liverpool, UK

Introduction In 2001 the National Institute for Clinical Excellence (NICE) published guidance on best practice for cancer services entitled Improving Outcomes Guidance (IOG). These guidelines specified centralisation, increased patient volume ( $>200$ referrals/ year), improved resection rates $(>10 \%-15 \%)$ and reduced postoperative mortality rates $(<5 \%)$. We looked at the development of a regional tertiary pancreatic centre over the 10 years following this, and how practice has changed over this time.

Methods A prospectively maintained database of all referrals with suspected pancreatic cancer to the Supra-Regional Pancreas Centre in Liverpool was interrogated to assess changes in practice and outcome from 2001 to 2010 inclusive. Data were analysed with $\chi^{2}$ for trend for categorical data and log rank for survival data.

Results 2076 patients with malignancy were referred, rising from 73 in 2001 to 364 in 2010. 511 resections for malignancy were performed (25\%), ranging between $21 \%(42 / 182)$ in 2005 and $36 \%$ (33/87) in 2003 per year, with no trend over time. 710 patients underwent planned operation for malignancy over the 10-year period ranging from 41 procedures in 2001 and peaking at 97 in 2008, with 94 procedures in 2009 and 88 cases in 2010. The percentage of planned resections that had a successful resection increased from 51\% (21/41) in 2001 to $90 \%$ (79/88) by 2010 $(\mathrm{p}<0.001)$. The mortality from resection was $9 / 567(2 \%)$ and overall was $5 \%(37 / 710)$ including palliative procedures. The 1 year survival rates of patients who underwent a successful resection improved from $65 \%(13 / 20)$ in 2001 to $76 \%(69 / 91)$ by $2009(p=0.02)$. There has been a rise in the number of intraductal papillary mucinous neoplasm (IPMN) resected with none in 2001 increasing to 12 resections during 2009, 10 in 2010 and 212 patients currently undergoing surveillance for IPMN. The number of staging laparoscopies has remained fairly constant at around 27 per year, despite the increase in referrals, which reflects more stringent criteria in selecting these patients for laparoscopy.

Conclusion Over the last 10 years we have seen centralisation of services, which was completed in 2007. This has led to an increase in volume of cancer referrals in line with IOG guidance. Although resection rates have stayed constant, this reflects the increasing number of patients referred with irresectable disease for other treatments, including chemotherapy and novel cancer trials. There has been an improvement in case selection as demonstrated by a reduction in the percentage of bypass procedures, reflecting better pre-operative staging of patients. This has also lead to an improved 1-year survival in those patients who had a successful resection.

Competing interests None declared.

\section{OC-058 INCREASED MORBIDITY IN OVERWEIGHT AND OBESE LIVER TRANSPLANT RECIPIENTS - A SINGLE CENTRE EXPERIENCE WITH 1325 PATIENTS}

doi:10.1136/gutjnl-2012-302514a.58

A Hakeem, ${ }^{*}$ A Cockbain, S Raza, S Pollard, G Toogood, M Attia, N Ahmad, E Hidalgo, K R Prasad, K Menon. Department of HPB and Liver Transplantation, St James's University Hospital NHS Trust, Leeds, UK

Introduction Obesity levels in the UK have risen over the years. Studies from the US and elsewhere have reported variable outcomes in terms of post liver transplant morbidity, mortality and graft survival in obese liver transplant recipients. There are no such reports from the UK. The aim of this study was to analyse the impact of BMI (Body Mass Index) on outcomes following adult liver transplantation.

Methods Data were retrieved from a prospectively maintained institutional database from 1994 to 2009. Patients were stratified into four BMI categories established by the WHO: underweight $\left(<18.5 \mathrm{~kg} / \mathrm{m}^{2}\right)$, normal weight $\left(18.5-<25.0 \mathrm{~kg} / \mathrm{m}^{2}\right)$, over weight $\left(>25.0-<30.0 \mathrm{~kg} / \mathrm{m}^{2}\right)$ and obese $\left(>30.0 \mathrm{~kg} / \mathrm{m}^{2}\right)$. Primary outcome was to evaluate post-operative morbidity and secondary measures were overall patient and graft survival. Categorical variables were analysed by $\chi^{2}$, and continuous variables by one-way ANOVA ( $p$ $<0.05$ was considered significant). Kaplan-Meier curves were used to study the effect of BMI categories on patient and graft survival. Results 1400 adult transplants were identified. 1325 patients had height and weight measurements and were included in the study. The overall morbidity was higher in overweight $(71.9 \%, \mathrm{p}<0.001)$ and obese patients $(69.2 \%, \mathrm{p}<0.001)$ in comparison to normal weight recipients $(64.3 \%)$. Post-operative septic events were common in overweight $(60.6 \%, \mathrm{p}=0.001)$ and obese patients $(61.0 \%$, $\mathrm{p}=0.007)$ in comparison to normal weight patients $(50.4 \%)$. Postoperative chest infections were much more common in obese $(14.2 \%$ vs $9.0 \%, p=0.038)$ and overweight recipients $(17.7 \%$ vs $9.0 \%$, p $<0.001)$ in comparison to normal weight recipients. Obese patients had significantly longer intensive care stay than normal weight patients (mean 4.1 vs 3.2 days, $p=0.043$ ). The length of post-operative hospital stay was significantly longer in obese (mean 21.5 days, $\mathrm{p}=0.009$ ) and overweight patients (mean 22.4 days, $\mathrm{p}=0.000$ ) in comparison to normal weight patients (mean 18.0 days). Similarly, ascitic or drain fluid sepsis was common in overweight patients in comparison to normal weight recipients $(16.5 \%$ vs $2.2 \%$, p < 0.001$)$. There was no difference in overall graft survival $(p=0.222)$ and patient survival $(p=0.196)$ between the four groups by log-rank test. Conclusion This is the largest and the only reported UK series on BMI and outcome following liver transplantation. Overweight and 
obese patients have increased morbidity in terms of septic complications following liver transplantation, with consequent increased length of intensive care and hospital stay. Identifying these patients early and introduction of measures to reduce BMI should be considered to improve outcomes following liver transplantation.

Competing interests None declared.

\section{OC-059 AN EXPERIMENTAL STUDY TO DETERMINE THE PATHOGENESIS OF OXALIPLATIN INDUCED SINUSOIDAL OBSTRUCTION SYNDROME}

doi:10.1136/gutjnl-2012-302514a.59

\begin{abstract}
${ }^{1,2} \mathrm{~S}$ Robinson, ${ }^{*}{ }^{2} \mathrm{~J}$ Mann, ${ }^{1} \mathrm{D}$ Manas, ${ }^{2} \mathrm{~A}$ Burt, ${ }^{2} \mathrm{D}$ Mann, ${ }^{1} \mathrm{~S}$ White. ${ }^{1} H P B$ \& Transplant Surgery, Freeman Hospital, Newcastle Upon Tyne, UK; ${ }^{2}$ Institute of Cellular Medicine, Newcastle University, Newcastle Upon Tyne, UK
\end{abstract}

Introduction Sinusoidal obstruction syndrome (SOS) following Oxaliplatin based chemotherapy is a cause for major concern when undertaking liver resection for colorectal liver metastases. To date no relevant experimental models of Oxaliplatin induced SOS have been described. The aim of this project was to establish such a model which could be utilised to identify potential therapeutic strategies to prevent the development of SOS.

Methods C57Bl/ 6 mice were treated with intra-peritoneal FOLFOX $(n=10)$, or vehicle $(n=10)$, weekly for 5 weeks and culled 1 week following final treatment. Representative biopsies of the liver and spleen were fixed in formalin and paraffin embedded for histological analysis. RNA and protein were extracted from snap frozen biopsies of the liver and subject to biochemical, analysis by qRT-PCR and western blot respectively, for markers of matrix remodelling, vascular dysfunction/endothelial damage, DNA damage and cellular proliferation. Serum was separated from whole blood and markers of liver injury (ie, ALT, AST and Alk Phos) were also measured. Statistical significance was assessed with Mann-Whitney U Test.

Results FOLFOX treatment was associated with the development of sinusoidal dilatation and peri-venular hepatocyte atrophy on H\&E stained sections of the liver in keeping with SOS. This was associated with an elevated serum ALT and AST $(p<0.05)$. Immunohistochemistry for $\gamma \mathrm{H} 2 \mathrm{AX}$ demonstrated the presence of DNA damage in the sinusoidal endothelium. In the liver of FOLFOX treated animals there was up-regulation of key genes associated with matrix remodelling such as MMP9 $(p<0.001)$, MMP2 $(p<0.001)$, ProCollagen I $(p<0.001)$ and TGF $\beta(p<0.001)$. There was evidence of endothelial damage and a subsequent pro-thrombotic state with upregulation of PAI-1 $(p<0.001)$, vWF $(p<0.01)$ and Factor $X(p<0.001)$. Conclusion We have developed the first reproducible model of chemotherapy induced SOS that reflects the pathogenesis of this disease process in patients. Through analysis of this model we have gained insights into the molecular changes that underpin the development of SOS and are now able to test potential therapeutic strategies to prevent it.

Competing interests None declared.

\section{OC-060 SURVIVAL AFTER DOWNSTAGING CHEMOTHERAPY FOR INITIALLY UN-RESECTABLE COLORECTAL LIVER METASTASES: EXPERIENCE FROM A UK RESECTION CENTRE}

doi:10.1136/gutjnl-2012-302514a.60

${ }^{1} \mathrm{G}$ R Irving, * 1 J P Tiernan, ' C D Briggs, 'M Peterson, ${ }^{2} \mathrm{C}$ Cameron. ${ }^{1} \mathrm{HPB}$ surgery, Sheffield Teaching Hospitals, Sheffield, UK; ${ }^{2} H P B$ surgery, Queens Medical Centre, Nottingham, UK

Introduction Liver metastases occur in $\sim 50 \%$ of patients with colorectal cancer. Only $20 \%$ of patients present with disease that is suitable for resection, the only hope of cure. In selected patients, chemotherapy may downstage inoperable liver-limited disease such that potentially curative resection becomes feasible.

Methods All patients referred to the regional Multi-Disciplinary Team (October 2001-June 2008) were considered for downstaging chemotherapy if they had inoperable liver-limited disease and were fit enough for resection. Two-weekly FOLFOX chemotherapy was administered and response assessed by 3 monthly CT scan. Disease having a partial response but remaining unresectable received further chemotherapy and reassessment by CT. Patients in whom R0 resection was thought feasible were offered surgery. Morbidity and mortality data were collected. Mortality was cross-referenced with the Cancer Registry. Overall (OS), post-operative (POS) and disease free survival (DFS) were calculated using SPSS (medians/ range/Kaplan-Meier survival curves). OS was calculated from the onset of chemotherapy and based on intention to treat. Additional univariate analysis has been performed.

Results 104 patients commenced downstaging chemotherapy (median six cycles): one died after the 3rd cycle (cardiac), 28 had no response, 56 had a degree of response and 19 had near complete regression. Eventually, 56 patients remained unresectable and 47 had a disease response deemed resectable and were offered surgery. Of these 47 patients, seven declined or became unfit for surgery and 40 proceeded to an operation, of which 36 underwent resection. In four, liver resection was abandoned due to additional disease found at surgery. Peri-operative morbidity was $63 \%$ and 30 -day mortality was zero. Mortality of patients receiving only chemotherapy was $100 \%$ and median OS 14 months (range 3-64 months) compared to a median OS for the 40 patients undergoing laparotomy of 39 months (10-98) with an estimated 20\% 5-year survival rate. The 10 surviving patients $(25 \%)$ have been followed up for a median of 63.5 months (36-90). 7/36 patients (19.4\%) remain disease free with median OS 85 months (40-98). $29 / 36$ patients (80.6\%) have recurred, all within 24 months of surgery (median DFS 7 months) but with median OS 35 months (10-73) and 4/29 (14\%) surviving $>5$ years.

Conclusion Liver resection after downstaging chemotherapy is safe, feasible and improves median survival. $80 \%$ of patients had recurring disease, all within 2 years, however a significant survival benefit occurred in this group compared to patients who could not be offered surgery. The proportion of patients remaining disease free $(19.4 \%)$ is lower than would be expected in a group with initially resectable disease.

Competing interests None declared.

\section{OC-061 GASTRIC ELECTRICAL STIMULATION FOR INTRACTABLE GASTROPARESIS}

doi:10.1136/gutjnl-2012-302514a.61

${ }^{1} \mathrm{~A}$ I Sarela, ${ }^{*} \mathrm{~S}$ Dexter, ${ }^{2} \mathrm{~F}$ Choudhury, ${ }^{3} \mathrm{M}$ Denyer. ${ }^{1}$ Upper Gl Surgery, St James's University Hospital, Leeds, UK; ${ }^{2}$ Nuclear Medicine, St James's University Hospital, Leeds, UK; ${ }^{3}$ Department of Gastroenterology, St James's University Hospital, Leeds, UK

Introduction Severe, drug-unresponsive gastroparesis is a debilitating condition. Treatment with gastric electrical stimulation using Enterra ${ }^{\circledR}$ was granted humanitarian device exemption status by the FDA in 2000. The largest case-series $(N=221)$ reports that $54 \%$ of patients had $>50 \%$ symptom-reduction with Enterra. ${ }^{1}$ We audited outcomes from Enterra therapy, with routine use of a trial of temporary stimulation to refine patient selection.

Methods Patients considered for Enterra during 2008-2011 were identified from a prospectively maintained database. Patients had been previously extensively investigated and treated for gastroparesis; $48 \%$ were referred by gastroenterologists and $37 \%$ by upper GI surgeons. Gastric emptying was assessed by scintigraphy in all 